\title{
STELLAR PARAMETERS IN THE BULGE CLUSTER NGC 6553
}

\author{
B. BARBUY \\ University of São Paulo, CP 9638, São Paulo 01065-970, Brazil \\ S. ORTOLANI \\ University of Padova, Vicolo dell'Osservatorio 5, I-35122 Padova, \\ Italy \\ E. BICA \\ University of Rio Grande do Sul, CP 15051, Porto Alegre \\ 91500-970, Brazil
}

AND

\begin{abstract}
A. RENZINI AND M.D. GUARNIERI
European Southern Observatory, Karl-Schwarzschild Str. 2, D85748 Garching bei München, Germany
\end{abstract}

\section{Introduction}

Globular clusters in the Galactic bulge form a flattened system, extending from the Galactic center to about $4.5 \mathrm{kpc}$ from the Sun (Barbuy et al. 1997). A study of abundance ratios in these clusters is very important for a more complete understanding of the bulge formation. In this work we present a spectroscopic analysis of individual stars in NGC 6553. This cluster is a key one because it is located at $\mathrm{d}_{\odot} \approx 5.1 \mathrm{kpc}$, therefore relatively close to us, and at the same time it is representative of the Galactic bulge stellar population: (a) Ortolani et al. (1995) showed that NGC 6553 and NGC 6528 show very similar Colour-Magnitude Diagrams (CMDs), and NGC 6528 is located at $\mathrm{d}_{\odot} \approx 7.83 \mathrm{kpc}$, very close to the Galactic center; (b) the stellar populations of the Baade Window is also very similar to that of NGC 6553 and NGC 6528 as Ortolani et al. (1995) have shown by comparing their luminosity functions.

The temperature calibration of metal-rich cool giants needs high quality photometric data. For giant stars in the metal-rich bulge globular cluster NGC6553 we have obtained JK photometry at ESO and VI photometry 
with the Hubble Space Telescope. This allowed us to derive effective temperatures. A detailed analysis of 3 stars was then carried out using échelle spectra obtained at the ESO $3.6 \mathrm{~m}$ telescope.

TABLE 1. Magnitudes

\begin{tabular}{lllll}
\hline star & V & I & J & K \\
III-3 & 15.825 & 13.408 & 11.536 & 10.328 \\
IV-13 & 15.586 & 13.114 & 11.282 & 10.028 \\
II-85 & 15.515 & 13.010 & 11.040 & 9.789 \\
\hline
\end{tabular}

TABLE 2. Derived temperatures

\begin{tabular}{llllll}
\hline star & T(V-I) & T(V-K) & T(J-K) & M(bol) & $\log g$ \\
\hline III-3 & 4021 & 3968 & 4220 & -2.26 & 0.95 \\
IV-13 & 3980 & 3944 & 4125 & -2.50 & 0.85 \\
II-85 & 3930 & 3880 & 4130 & -2.57 & 0.80 \\
\hline
\end{tabular}

\section{Stellar Parameters}

\subsection{TEMPERATURES}

The crucial issue in the detailed analysis of these cool and metal-rich giants is the determination of their effective temperatures $T_{\text {eff }}$. We succeeded to obtain very high quality $\mathrm{V}$ and I colours using the Hubble Space Telescope (HST) (Ortolani et al. 1995), and J and K colours using the detector IRAC2 at the $2.2 \mathrm{~m}$ telescope of ESO (Guarnieri et al. 1997). In Table 1 are given the magnitudes. The calibration of our old ESO Danish telescope data (Ortolani et al. 1990) showed an offset of about $0.28 \mathrm{mag}$, very probably due to crowding.

A further step in the derivation of colours is the reddening value adopted. In Guarnieri et al. (1997) we derived a colour excess of $E(V-I)=0.95$ for NGC 6553. Assuming a ratio $E(V-I) / E(B-V)=1.35$ (Terndrup 1988; Grebel \& Roberts 1995) the resulting $E(B-V)$ is 0.7. Assuming $\mathrm{E}(\mathrm{V}-\mathrm{K}) / \mathrm{E}(\mathrm{B}-\mathrm{V})=2.744$ and $\mathrm{E}(\mathrm{J}-\mathrm{K}) / \mathrm{E}(\mathrm{B}-\mathrm{V})=0.527$ (Rieke \& Lebofsky $1983)$, and $\mathrm{E}(\mathrm{V}-\mathrm{I})_{C} / \mathrm{E}(\mathrm{B}-\mathrm{V})=1.33$ (Dean et al. 1978) the available colours 
were de-reddened.

In order to derive temperatures we used several methods:

(i) Infrared flux method: Based on absolute measurements of stellar monochromatic fluxes in the infrared region for a sample of 80 solar metallicity stars, Blackwell \& Lynas-Gray (1994) established the relation $\mathrm{T}(\mathrm{V}-\mathrm{K})=8862$ $2583(\mathrm{~V}-\mathrm{K})-353.1(\mathrm{~V}-\mathrm{K})^{2}$.

(ii) Relations by McWilliam (1990): Based on a sample of 671 giants of about solar metallicity McWilliam (1990) derived the following relations: $\mathrm{T}(\mathrm{B}-\mathrm{V})=8351-4936(\mathrm{~B}-\mathrm{V})+1456(\mathrm{~B}-\mathrm{V})^{2}-78(\mathrm{~B}-\mathrm{V})^{2} ; \mathrm{T}(\mathrm{V}-\mathrm{K})=8595-$ $2349(\mathrm{~V}-\mathrm{K})+321(\mathrm{~V}-\mathrm{K})^{2}-8(\mathrm{~V}-\mathrm{K})^{2} ; \mathrm{T}(\mathrm{V}-\mathrm{I})=9853-6733(\mathrm{~V}-\mathrm{I})+2564(\mathrm{~V}-$ $\mathrm{I})^{2}-335(\mathrm{~V}-\mathrm{I})^{2} . \mathrm{T}(\mathrm{J}-\mathrm{K})=8176-8715(\mathrm{~J}-\mathrm{K})+6850(\mathrm{~J}-\mathrm{K})^{2}-2489(\mathrm{~J}-\mathrm{K})^{2}$.

(iii) Tables by Bessell et al. (1996): Using Bessell et al. (1996) calibrations based on NMARCS models (Plez et al. 1992), give the temperatures reported in Table 2, which correspond essentially to $\mathrm{T}_{\text {eff }}=3950 \mathrm{~K}$ for the three sample stars.

The final adopted temperatures for our stars are those corresponding to the Bessell et al. calibration of (V-I) and (V-K) colours.

\subsection{GRAVITIES}

The classical relation $\log g_{*}=4.44+4 \log T_{*} / T_{\odot}+0.4\left(M_{\text {bol }}-4.67\right)+$ $\log M_{*} / M_{\odot}$ was used, where $\mathrm{T}_{\odot}=5770 \mathrm{~K}, \mathrm{M}_{*}=0.8 \mathrm{M}_{\odot}, \mathrm{M}_{\mathrm{bol} \odot}=4.67$ were adopted; for deriving $\mathrm{M}_{\mathrm{bol} *}$ we adopted $\mathrm{M}_{\mathrm{V}}(\mathrm{HB})=1.06$ (Buonanno et al. 1989), $\mathrm{V}(\mathrm{HB})=15.8$ (Ortolani et al. 1995) and bolometric magnitude corrections by Persson et al. (1980), and the resulting $\mathrm{M}_{\mathrm{bol}}$ are given in Table 2 .

\subsection{METALLICITY}

The metallicities were derived by plotting curves of growth of FeI, where the equivalent widths where a selected list of lines were measured using IRAF, and the code RENOIR by M. Spite was employed for plotting the curves of growth. The resulting metallicity for the cluster is $[\mathrm{Fe} / \mathrm{H}] \approx-0.35$.

\section{Average final abundance ratios}

We find the preliminary abundance ratios: $[\mathrm{Mg} / \mathrm{Fe}] \approx+0.15,[\mathrm{Ti} / \mathrm{Fe}] \approx$ $+0.3,[\mathrm{Si} / \mathrm{Fe}] \approx+0.6,[\mathrm{Ca} / \mathrm{Fe}] \approx 0.0$ and $[\mathrm{Eu} / \mathrm{Fe}] \approx+0.3$.

It is interesting to compare these results with abundance ratios for the bulge from the literature. McWilliam \& Rich (1994) analyzed 11 Baade Window $\mathrm{K}$ giants, for which they obtained: $[\mathrm{Mg} / \mathrm{Fe}] \approx[\mathrm{Ti} / \mathrm{Fe}] \approx+0.3$ and $[\mathrm{Ca} / \mathrm{Fe}] \approx[\mathrm{Si} / \mathrm{Fe}] \approx 0.0$. Idiart, Freitas Pacheco \& Costa (1996) derived from and integrated spectrum of the Baade Window $[\mathrm{Mg} / \mathrm{Fe}] \approx+0.45$. 
Terndrup et al. (1995), Sadler et al. (1996) used medium-resolution spectra of 400 giants in the Baade Window for which they obtained $[\mathrm{Mg} / \mathrm{Fe}] \approx$ $0.2-0.3$. It is also interesting to point out that all these results are also in agreement with $\mathrm{Mg}$-to-Fe ratios in bulges of ellipticals: Worthey et al. (1992), Bender (1996), Barbuy et al. (1996) presented evidences pointing to enhanced $[\mathrm{Mg} / \mathrm{Fe}]$ in bulges of ellipticals.

\section{References:}

Barbuy, B., Bica, E., Ortolani, S.: 1997, A\&A, submitted

Barbuy, B., Castro, S., Ortolani, S., Bica, E.: 1992, A\&A, 259,607

Barbuy, B., Freitas Pacheco, J.A., Idiart, T.: 1996, in IAU S.

171, New Light on Galaxy Evolution, Kluwer, eds. R. Bender,

R. Davies, p. 340

Bender, R.: 1996, 1996, in IAU S. 171, New Light on Galaxy

Evolution, Kluwer, eds. R. Bender, R. Davies, p. 181

Bessell, M.S., Castelli, F., Plez, B.: 1996, preprint

Blackwell, D.E., Lynas-Gray, A.E.: 1994, A\&A, 282, 899

Buonanno, R., Corsi, C.E., Fusi-Pecci, F.: 1989, A\&A, 216, 80

Dean, J., Warpen, P., Cousins, A.: 1978, MNRAS, 183, 569

Grebel, A.K., Roberts, W.: 1995, A\&AS, 109, 293

Guarnieri, M.D., Ortolani, S., Montegriffo, P., Renzini, A.,

Barbuy, B., Bica, E., Moneti, A.: 1997, A\&A, in press

Idiart, T., Freitas Pacheco, J.A., Costa, R.D.D.: 1996, AJ, 111,1169

McWilliam, A.: 1990, ApJS, 74, 1075

McWilliam, A., Rich, R.M.: 1994, ApJS, 91, 749

Ortolani, S., Barbuy, B., Bica, E.: 1990, A\&A, 236, 362

Ortolani, S., Renzini, A., Gilmozzi, R., Marconi, G., Barbuy,

B., Bica, E., Rich, R.M.: 1995, Nature, 377, 701

Persson, S.E., Frogel, J.A., Cohen, J.G., Aaronson, M.,

Matthews, K.: 1980, ApJ, 235, 452

Plez, B., Brett, J.M., Nordlund, A.: 1992, A\&A, 256, 551

Rieke, G.H., Lebofsky, M.J.: 1985, ApJ, 288, 618

Sadler. E.M., Terndrup, D.M., Rich, R.M.: 1996, AJ, 112, 117

Terndrup, D.M.: 1988, AJ, 96,884

Terndrup, D.M., Sadler, E.M., Rich, R.M.: 1995, AJ, 110, 1774

Worthey, G., Faber, S.M., Gonzalez, J.J.: 1992, ApJ, 398, 69

\section{DISCUSSION}

SHETRONE: Are your results not similar to the Edvardsson et al. work for disk stars? That is, when $\mathrm{Mg}$ is slightly enhanced, $\mathrm{Ti}$ is significantly enhanced, regardless of $\mathrm{Ca}$. The same pattern is repeated in this cluster.

BEATRIZ BARBUY: I believe these clusters are not a typical disk population like the stars studied by Edvardsson et al., but a typ bulge population. As a matter of fact, the bulge shows a mean met. of $[\mathrm{Fe} / \mathrm{H}] \simeq-0.2$ (McWilliam \& Rich 1994, ApJS, 91, 749). 